\title{
Hadronic triggers and trigger object-level analysis at ATLAS
}

\section{Donatas Ramilas Zaripovas*}

The University of Glasgow

E-mail: donatas.ramilas.zaripovas@cern.ch

Hadronic signatures are critical to the high energy physics analysis program at the Large Hadron Collider (LHC), and are broadly used for both Standard Model measurements and searches for new physics. These signatures include generic quark and gluon jets, as well as jets originating from $b$-quarks or the decay of massive particles (such as electroweak bosons or top quarks). Additionally missing transverse momentum from non-interacting particles provides an interesting probe in the search for new physics beyond the Standard Model. Developing trigger selections that target these events is a huge challenge at the LHC due to the enormous event rates associated with these signatures. This challenge is exacerbated by the amount of pileup activity, which continues to grow. In order to address these challenges, several new techniques have been developed during the past year in order to significantly improve the potential of the 2017 dataset and overcome the limiting factors, such as storage and computing requirements for the analysis of this data.

This article presents an overview of how hadronic signatures are triggered at the ATLAS experiment, outlining the challenges of hadronic object triggering and describing the improvements performed over the course of the Run 2 LHC data-taking program, such as analysis of a compact data stream involving trigger-level objects, recorded at a higher rate than is possible for full event data. The performance in Run 2 data will be shown, including demonstrations of the new techniques being used in 2017. We also discuss further critical developments envisaged for the rest of Run 2.

The European Physical Society Conference on High Energy Physics

5-12 July, 2017

Venice

\footnotetext{
* Speaker.

${ }^{\dagger}$ On behalf of the ATLAS Collaboration.
} 


\section{LHC challenges and the ATLAS trigger}

On 29 April, just after 8.00 p.m., the Large Hadron Collider (LHC) began circulating beams for the first time in 2017 after its technical shutdown from December 2016, during which a number of improvements took place to ensure a smooth transition into the second phase of Run 2. During this phase the LHC is forecast to reach the maximum Run 2 instantaneous luminosity conditions of up to $2 \times 10^{34} \mathrm{~cm}^{-2} \mathrm{~s}^{-1}$ and in doing so will deliver increasing pileup rates in the new data taking period. To maintain or even improve the Run 2 physics potential of ATLAS in such a harsh environment it was essential to improve trigger performance - without improvement, trigger efficiencies would drop to a level that significantly deteriorates physics sensitivity.

The ATLAS trigger [1] is a system that makes the decision of whether or not to record a particular collision event in the ATLAS detector [2]. The readout rate of the ATLAS detector is limited to about $1 \mathrm{kHz}$ while the average beam crossing rate of the $\mathrm{LHC}$ is $\sim 30 \mathrm{MHz}$, this means that only the most interesting events can be kept. The ATLAS trigger therefore has crucial impact on the quality of data used in physics analysis. The ATLAS trigger system reduces the rate in two steps, firstly a hardware based trigger (Level 1 (L1)) and, secondly, a software based High Level Trigger (HLT).

\section{Improvements in small- $R$ jets}

In 2016, jets were calibrated at the HLT based on offline calibrations derived from data collected in 2012. In 2017, the calibration was updated to use data collected in 2015 and 2016, and was additionally altered to use more steps from the offline calibration chain to improve agreement between offline and HLT jets. The extra jet trigger calibration steps of 2017 include the Global Sequential Calibration (GSC) corrections [3] and the application of in-situ corrections [4]. GSC corrects jets according to their longitudinal shower shape and associated track characteristics without changing the overall energy scale. The procedure can be split into parts involving calorimeter-based variables, and parts involving track-based variables. Calorimeter-only GSC procedure became a default calibration step in 2017, but with full track reconstruction currently being too costly to run at the HLT for every event, the track-based part of the GSC is currently only run for a subset of available triggers. A significant improvement has been observed in triggers that use a combination of calorimeter and track-based GSC as is shown in Figure 1, where a higher efficiency is achieved at lower $p_{\mathrm{T}}$ thresholds using the updated calibration technique. Furthermore, the two in-situ calibrations that became the default calibrations for HLT jets in 2017 are known as the $\eta$-intercalibration, the purpose of which is to make the data-MC response uniform across the entire $\eta$ range, and the in-situ Jet Energy Scale (JES) correction, which corrects the resulting jet energy in all regions across the $\eta$ range by factors derived in $|\eta|<0.8$ [5]. Together, these additional corrections allow for improved agreement between the scale of trigger and offline jets as a function of both $\eta$ and $p_{\mathrm{T}}$, and thus the trigger efficiency rises much more rapidly. 

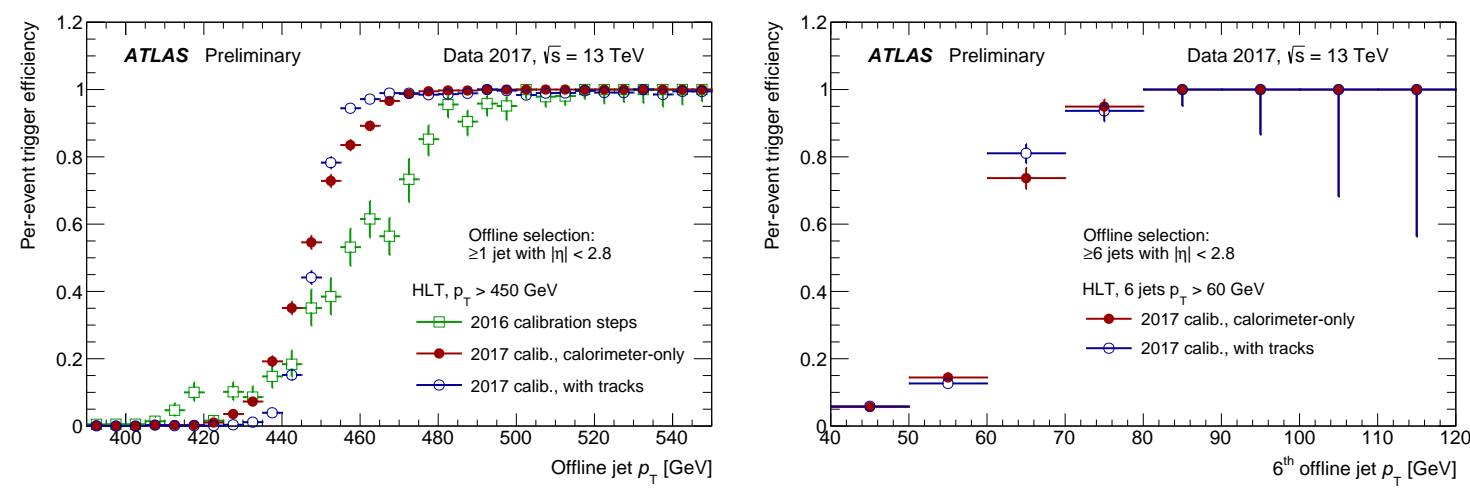

(a) Single-jet trigger efficiency for HLT jets

(b) Multi-jet trigger efficiency for HLT jets with $p_{\mathrm{T}}>450 \mathrm{GeV}$.

with $p_{\mathrm{T}}>60 \mathrm{GeV}$ and $>6$ jets.

Figure 1: Per-event trigger efficiencies for (a) a single jet trigger and (b) a multi-jet trigger with three (two) different calibration options applied. In red (closed circles) is the updated calibration applied in 2017, utilising only calorimeter information, and in blue (open circles) is the updated calibration additionally with track information. Calibration steps taken in 2016 are also shown in

(a) (green open squares) [6].
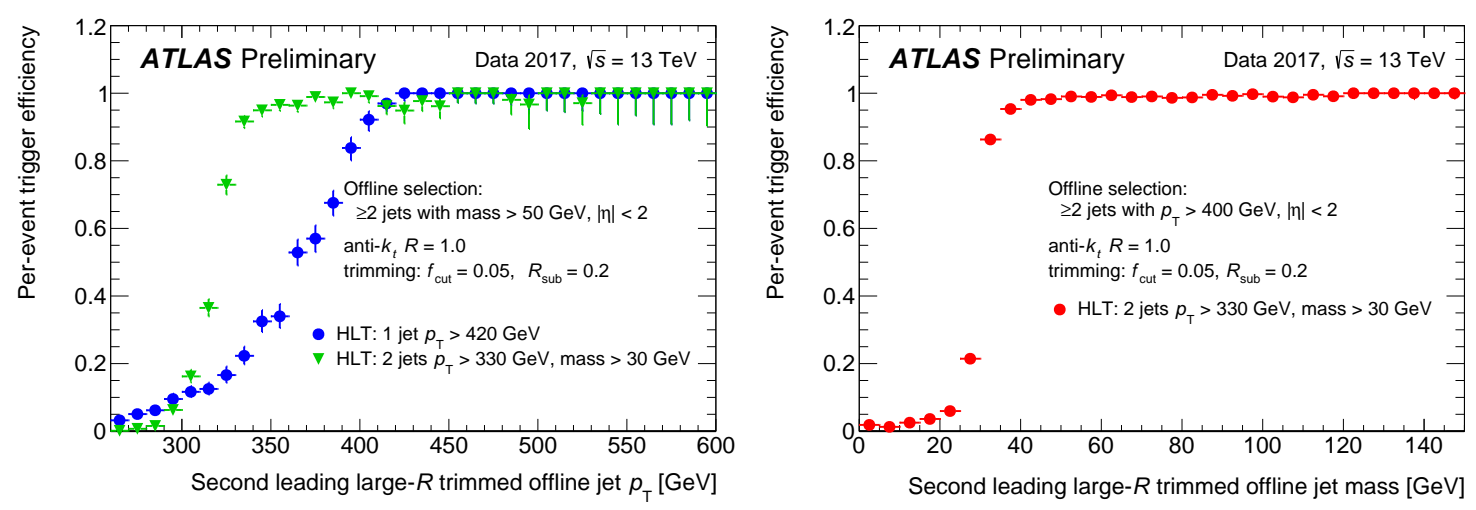

(a) Blue circles represent a trimmed large- $R$ single- (b) Red circles represent a trimmed large- $R$ di-jet jet trigger with $p_{\mathrm{T}}>420 \mathrm{GeV}$. An additional $30 \mathrm{GeV}$ trigger with $p_{\mathrm{T}}>330 \mathrm{GeV}$ and an additional $30 \mathrm{GeV}$ cut on the leading and second leading jet masses is cut on the jet masses of the leading and second leadshown in green triangles. ing selected trimmed trigger jets.

Figure 2: Efficiencies for HLT large- $R$ triggers are shown as a function of the second leading offline trimmed jet $p_{\mathrm{T}}$ for jets with $|\eta|<2.0$ and jet mass above $50 \mathrm{GeV}$ (a) and jet $p_{\mathrm{T}}$ above $400 \mathrm{GeV}$ (b) $[6]$.

\section{Improvements in large- $R$ jets}

In the kinematic regime that the LHC is operating at, it is possible to produce heavy bosons and top quarks with a transverse momentum such that it considerably exceeds their rest mass. Thus their decay products are in turn collimated, and (if hadronic) merge into what are known as boosted jets with large radius (large- $R$ ) - a single collimated spray of hadrons with a large angular 
separation and with substructure originating from the initial $W / Z /$ top decay products. Since they cover a substantially larger area of the detector than small- $R$ jets, large- $R$ jets are more susceptible to pileup, and therefore it is important to employ jet substructure techniques to mitigate this effect and improve the efficiency of boosted jet triggers. A method known as trimming was shown to reduce the effects of pileup by recursively removing soft narrow $(R=0.2)$ jets from the original jet, leaving only high energy density spots [7]. The resulting efficiency curves can be seen in Figure 2. A fluctuation in efficiency in the respective plateau regions can be observed in both triggers due to small differences between the application of the trimming procedure to trigger and offline jets. To mitigate this effect, the trimming parameter $f_{\text {cut }}$ of 0.04 will be used for trigger jets, while the standard offline selection uses $f_{\text {cut }}=0.05$. Once a pileup-stable jet collection is achieved, cuts on the invariant mass of the reconstructed jet can be used to significantly lower thresholds for a fixed rate, as can be seen in Figure 2. They work to significantly suppress the QCD di-jet background, allowing a much lower $p_{\mathrm{T}}$ threshold of $330 \mathrm{GeV}$ in the case of Figure 2(a), while retaining nearly all signal-like jets with a mass of above $50 \mathrm{GeV}$. These options have been implemented and are available for large- $R$ jet collections as of 2017.

\section{Trigger-object Level Analysis in ATLAS}

The purpose of working towards increasing the luminosity without ramping up the center of mass energy at the LHC is to increase the statistical sample size that many analyses rely on to increase the sensitivity to potential new physics. Currently and as in the case of recent results from ATLAS, the main focus is on mass regions above $1 \mathrm{TeV}$ [8], even though the lower mass regions are still very interesting for mass resonances with lower cross-sections. However, accessing these sub-TeV regions at the LHC is challenging due to a large Standard Model background that, combined with a limited bandwidth of inclusive single-jet triggers, severely limits the statistical power required for these searches. A procedure known as prescaling is used to control the trigger output rate, where only a fraction of events are retained for analysis, and is one of the main reasons for loss of sensitivity in lower $p_{\mathrm{T}}$ regions. To overcome this limitation a technique called Triggerobject Level Analysis (TLA) can be adopted, where only the event information (HLT objects) relevant for a particular physics analysis is recorded. This reduces the size of the event being recorded and thus the event rate can be increased substantially while having a small impact on overall bandwidth. Figure 3(a) highlights the increase in statistics achieved by the TLA strategy as compared to selecting any single jet trigger in a region where these triggers are normally heavily prescaled, while the impact on the overall bandwidth (see Figure 3(b)) can be kept below $1 \%$.

This technique was used for the first time in ATLAS [10] to set limits on light di-jet resonances and is currently offering a novel way of approaching searches for new physics in the low-mass regime.

\section{Conclusions and prospects}

While 2016 has been a very successful year of data taking, 2017 is a challenging year for the ATLAS trigger due to the need to cope with the high luminosity running conditions of Run 2 whilst building on ATLAS physics potential. Improvements in jet since 2016 have allowed us 


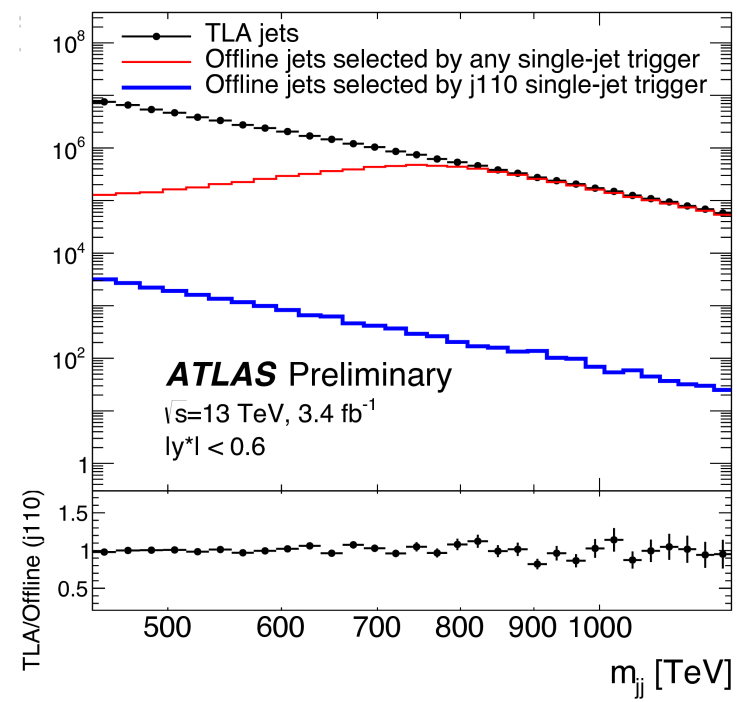

(a) Comparison between the data used by the Trigger- in July 2016.

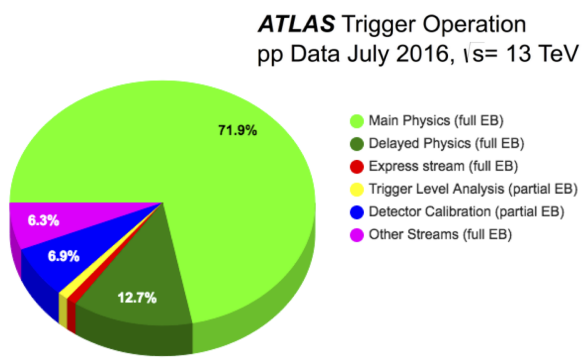

(b) Contributions to the total output bandwidth from the various streams at the ATLAS HLT for a fill taken

object Level Analysis and the data selected by using an OR of any single jet trigger.

Figure 3: TLA performance and impact on the total bandwidth of the ATLAS HLT [9].

to substantially improve the jet trigger efficiency but work is still ongoing: implementation of the Fast TracKer (FTK) [11] may allow all jet triggers to benefit from the improved energy resolution offered by the track-based GSC, reducing the rate allocated to low $p_{\mathrm{T}}$ threshold support triggers and thus allowing us to record a larger fraction of high $p_{\mathrm{T}}$ threshold events for physics analyses. In addition to this, improved jet trigger resolution will consequently improve the power of the TLA technique, paving the way for a better understanding of physics in the sub-TeV kinematic regime. While the implementation of jet substructure techniques has shown great potential for improvement in large- $R$ triggers that are suffering from high-pileup conditions, more variables are being implemented for large- $R$ jets that are expected to further suppress the QCD di-jet background. Moreover, ATLAS has recently commissioned a new component of the Level 1 trigger, the L1 Topological (L1Topo) trigger module [12], this enables promising alternative L1 items which will allow more efficient selection of large- $R$ jets with many subjets, while also allowing TLA to save rate through the use of L1Topo $\Delta \eta$ cuts.

\section{References}

[1] ATLAS Collaboration, Performance of the ATLAS Trigger System in 2015, Eur. Phys. J. C77 (2017) 317, arXiv:1611.09661 [hep-ex].

[2] ATLAS Collaboration, The ATLAS Experiment at the CERN Large Hadron Collider, JINST 3 (2008) S08003.

[3] ATLAS Collaboration, Jet global sequential corrections with the ATLAS detector in proton-proton collisions at $\sqrt{s}=8 \mathrm{TeV}$, ATLAS-CONF-2015-002,

URL: https://cds.cern.ch/record/2001682. 
[4] ATLAS Collaboration, Jet Calibration and Systematic Uncertainties for Jets Reconstructed in the ATLAS Detector at $\sqrt{s}=13 \mathrm{TeV}$, ATL-PHYS-PUB-2015-015, URL: https://cds.cern.ch/record/2037613.

[5] ATLAS Collaboration, Data-driven determination of the energy scale and resolution of jets reconstructed in the ATLAS calorimeters using dijet and multijet events at $\sqrt{\mathrm{s}}=8 \mathrm{TeV}$, ATLAS-CONF-2015-017, URL: https://cds.cern.ch/record/2008678.

[6] C. W. Kalderon, A. Martyniuk, N. Sherafati, and D. R. Zaripovas, Jet Trigger Plots for EPS 2017, ATL-COM-DAQ-2017-063, URL: https://cds.cern.ch/record/2271959.

[7] D. Krohn, J. Thaler, and L.-T. Wang, Jet Trimming, JHEP 02 (2010) 084, arXiv:0912.1342 [hep-ph].

[8] ATLAS Collaboration, Search for new phenomena in a lepton plus high jet multiplicity final state with the ATLAS experiment using $\sqrt{s}=13$ TeV proton-proton collision data, JHEP 09 (2017) 088, arXiv:1704.08493 [hep-ex].

[9] ATLAS Collaboration, URL: https://twiki.cern.ch/twiki/bin/view/AtlasPublic/TriggerOperationPublicResults Accessed: 2017-10-04.

[10] ATLAS Collaboration, Search for light dijet resonances with the ATLAS detector using a Trigger-Level Analysis in LHC pp collisions at $\sqrt{s}=13 \mathrm{TeV}$, ATLAS-CONF-2016-030, URL: https://cds.cern.ch/record/2161135.

[11] S. Citraro, A. Annovi, N. Biesuz, P. Giannetti, P. Luciano, H. Nasimi, M. Piendibene, C. L. Sotiropoulou, and G. Volpi, Highly Parallelized Pattern Matching Hardware for Fast Tracking at Hadron Colliders, IEEE Trans. Nucl. Sci. 63 (2016) 1147-1154.

[12] S. Artz et al., The ATLAS Level-1 Muon Topological Trigger Information for Run 2 of the LHC, JINST 10 (2015) C02027. 